\title{
Formación de la perspectiva científica de los estudiantes para la implementación de actividades profesionales
}

\section{Formation of the students' scientific outlook for the implementation of professional activities}

\author{
Olga N. Soluyanova \\ Moscow State University of Civil Engineering, Moscow, Russia. \\ ORCID: https://orcid.org/0000-0003-4085-1347 \\ Elena M. Voronova \\ Togliatti State University, Togliatti, Russia. \\ ORCID: https://orcid.org/0000-0002-0194-1289

\section{Svetlana V. Sergeeva} \\ Penza State Technological University, Penza, Russia. \\ ORCID: https://orcid.org/0000-0002-3612-3112

\section{Zhanna V. Smirnova} \\ Minin Nizhny Novgorod State Pedagogical University, Nizhny Novgorod, Russia. \\ ORCID: https://orcid.org/0000-0001-9950-9824
}

\section{Alla K. Milyaeva}

Moscow Region State University, Mytishchi, Russia. ORCID: https://orcid.org/0000-0003-4393-2951

*Correspondence

Email: ivannovadremova29@gmail.com

\section{Cite as:}

Soluyanova, O.N., Voronova, E.M., Sergeeva, S.V.,
Smirnova, Z.V., Milyaeva, A.K. (2021). Formación de la
perspectiva científica de los estudiantes para la
implementación de actividades profesionales. Propósitos
$y \quad$ Representaciones, 9 (SPE1), e922. Doi:
http://dx.doi.org/10.20511/pyr2021.v9nSPE1.922

Soluyanova, O.N., Voronova, E.M., Sergeeva, S.V., Smirnova, Z.V., Milyaeva, A.K. (2021). Formación de la implementación de actividades profesionales. Propósitos http://dx.doi.org/10.20511/pyr2021.v9nSPE1.922 


\section{Resumen}

La educación profesional moderna tiene como objetivo formar especialistas creativos altamente calificados capaces de resolver rápidamente los problemas profesionales. La resolución de este problema depende de muchos factores interrelacionados, entre los que ocupa un lugar significativo la formación de la cosmovisión científica de los estudiantes. El trabajo tiene como objetivo identificar las características de la formación y desarrollo de la cosmovisión científica de los estudiantes para la implementación de futuras actividades profesionales. El artículo presenta la experiencia de implementar tecnologías educativas modernas que contribuyan a la formación de la capacidad de los estudiantes para utilizar los fundamentos del conocimiento filosófico y socio-humanitario. Sobre el ejemplo de algunas disciplinas señaladas en el currículo, se revelan los métodos y formas del desarrollo de la cosmovisión científica. Las tecnologías, métodos y formas resaltados permiten crear las condiciones para unir a los estudiantes en grupos de proyectos para participar en diversas competencias profesionales que amplían las posiciones científicas y de cosmovisión. Para identificar el nivel de la cosmovisión científica, hemos identificado los criterios e indicadores apropiados. La dinámica observada muestra un aumento en el número de participantes en las competencias y un aumento en el nivel de formación de la cosmovisión científica. Los resultados del estudio permiten decir que la formación de la visión del mundo científico amplía las posibilidades de los estudiantes en la implementación de actividades profesionales.

Palabras clave: competencia profesional, formación profesional, visión científica, actividades de investigación.

\section{Summary}

Modern professional education is aimed at training highly qualified creative specialists capable of promptly solving professional problems. The resolution of this issue depends on many interrelated factors, among which the formation of the scientific worldview of students occupies a significant place. The work aims is to identify the features of the formation and development of the scientific worldview of students for the implementation of future professional activities. The article presents the experience of implementing modern educational technologies that contribute to the formation of students' ability to use the foundations of philosophical and sociohumanitarian knowledge. On the example of some disciplines indicated in the curriculum, the methods and forms of the development of the scientific worldview are revealed. The highlighted technologies, methods and forms make it possible to create conditions for uniting students into project groups to participate in various professional competitions that expand scientific and worldview positions. To identify the level of the scientific worldview, we have identified the appropriate criteria and indicators. The observed dynamics shows an increase in the number of participants in competitions and an increase in the level of formation of the scientific worldview. The results of the study allow us to say that the formation of the scientific world outlook expands the possibilities of students in the implementation of professional activities.

Key Words: professional competence, professional education, scientific outlook, research activities.

\section{Introducción}

Las necesidades cambiantes del Estado y la sociedad han actualizado la implementación de procesos de reforma en el campo de la educación vocacional, que ha identificado nuevos objetivos educativos. El proceso de aprendizaje en las escuelas superiores modernas tiene como objetivo desarrollar la competencia profesional de los estudiantes, que incluye muchos factores diferentes. La perspectiva científica del estudiante juega un papel importante en la formación de la competencia profesional (Donetskova, 2019). El estándar educativo del estado federal establece la necesidad que los estudiantes desarrollen competencias clave generales, incluida la capacidad 
de utilizar los fundamentos del conocimiento filosófico y socio-humanitario para formar una cosmovisión científica (Lutfullaev et al., 2020). El sistema doméstico moderno de educación superior se rige por las exigencias de una sociedad postindustrial, que presupone un alto nivel de movilidad estudiantil, su disposición a la autoeducación y la superación profesional (ArbeláezCampillo et al., 2020). La formación de la cosmovisión científica puede cambiar cualitativamente la implementación de la futura actividad profesional de los estudiantes (Chertovskikh, 2019).

Para los estudiantes modernos, la preparación para el autogobierno, las actividades de búsqueda y el desarrollo independiente de información adicional es importante, por lo tanto, las instituciones de educación superior están buscando formas innovadoras de formar una cosmovisión científica que contribuya al logro de una actitud consciente hacia las futuras actividades profesionales y evaluación de su papel en la sociedad (Orlova et al., 2018).

La transición del conocimiento existente a las convicciones ideológicas en la edad del estudiante ocurre solo en el proceso de desarrollar la capacidad de analizar la situación de forma independiente y sacar conclusions (Eliseeva et al., 2020). Las creencias se forman en el proceso de profundizar en la esencia de las ideas de cosmovisión. Sin embargo, si los estudiantes dominan las ideas de la cosmovisión, esto no significa que el proceso de formación de creencias esté completo (Yakovleva, 2019). El trabajo del maestro es animar a los estudiantes a utilizar estas ideas para que se conviertan en sus creencias (Kalinkina et al., 2017).

La cosmovisión de una persona es una colección de elementos separados e interconectados. En combinación, forman cualidades integrales de cosmovisión. La cosmovisión del estudiante es dinámica, sujeta a cambios y se revela en el proceso de interacción educativa, la formación de la competencia profesional, la solución de cualquier problema, discusiones, por lo tanto, es importante que el docente corrija oportunamente las acciones de los estudiantes para crear un entorno favorable (Filchenkova, 2019).

La forma activa de la cosmovisión científica de los estudiantes se lleva a cabo en el proceso de actividad independiente (con el apoyo correctivo del docente) (Grigoriev et al., 2019). En el proceso de realización de proyectos grupales e individuales, resolución de casos, discusiones, participación en juegos, los estudiantes forman sus propios puntos de vista, correlacionándolos con ideas y posiciones existentes (Eliseeva et al., 2020).

El éxito de la formación de una cosmovisión científica se logra al influir en las esferas intelectual, emocional, volitiva y motivacional de los estudiantes.

En la actividad profesional de un docente, la formación de las posiciones ideológicas de los estudiantes es uno de los procesos más complejos y multifacéticos. Cabe decir que las peculiaridades de la cosmovisión de los jóvenes son inconsistentes e inconsistentes. Por lo tanto, en el curso de la formación de la competencia profesional, es importante estudiar oportunamente el nivel de la cosmovisión científica de los estudiantes y someterlo a corrección.

\section{Marco teórico}

El análisis de la literatura científica nos permite hablar de los fundamentos de la formación de la cosmovisión científica de los estudiantes como una de las cuestiones más urgentes y tareas primarias resueltas en la pedagogía profesional moderna (Kosikhina, 1985). Sin embargo, la mayoría de los estudios no dan a conocer plenamente el tema de la formación de la cosmovisión científica de los estudiantes de instituciones de educación superior para la implementación de actividades profesionales, por lo que se requiere un estudio adicional de esta área (Petrichev et al., 2018). 
La teoría y metodología de la cosmovisión científica se revelan en los trabajos de G.M. Andreeva, L.I. Bozovic, N.K. Krupskaya. M.N. hizo una gran contribución al desarrollo de la cosmovisión científica. Pokrovsky, G.V. Platonov, I.D. Zverev y otros.

En el proceso de formar una cosmovisión científica, nos basamos en su comprensión como uno de los tipos de cosmovisión de la personalidad del estudiante (Birzhenyuk et al., 2020). La base de la cosmovisión científica es el sistema de conocimientos e ideas filosóficos, científicos fundamentales y científicos generales. N.K. Barsukova y T.I. Shishelova, como condiciones pedagógicas que inciden directamente en el resultado de la formación de la cosmovisión científica de los estudiantes, distinguen la implementación de programas para la formación de los fundamentos de la cosmovisión científica; estímulo de los estudiantes a actividades de aprendizaje independiente destinadas a expandir la cosmovisión científica; evaluación de la efectividad de la formación de una cosmovisión científica, corrección de los resultados (Barsukova et al., 2008).

La formación de una cosmovisión científica se considera como un proceso holístico en el que los estudiantes se involucran gradualmente en la actividad cognitiva, lo que les permite desarrollar visiones científicas sobre el orden mundial y la actividad profesional en el mundo modern (Pinkovetskaia et al., 2020). La cosmovisión científica puede revelarse como una formación compleja y multifacética del mundo interior de una persona, basada en el conocimiento científico generalizado sobre la imagen científica del mundo (Ivanov et al., 2020).

E.A. Veselova cree que el campo para el desarrollo de la visión científica de los estudiantes es la educación de una persona que sea capaz de resolver problemas socioeconómicos complejos a partir de nuevos significados de la vida (Tishchenko, 2020). Al mismo tiempo, el contenido del conocimiento se construye a partir de ideas de cosmovisión que contribuyen al desarrollo de competencias profesionales (Veselova, 2008).

Según Yu.V. Maslennikova e I.V. Grebenev, la base de la actividad, un medio para formar una cosmovisión científica es el contenido y la metodología para estudiar los fundamentos de las disciplinas relevantes, que se adapta a áreas específicas de estudio (Maslennikova et al., 2013).

\section{Metodología}

En la investigación participaron 142 estudiantes de instituciones de educación superior. Hemos estudiado la dinámica de la formación de la cosmovisión científica de los estudiantes durante tres años.

Para determinar la formación de una cosmovisión científica se identificaron niveles (alto, medio, bajo) y criterios: el grado de dominio de conceptos científicos, aparato terminológico, que son de gran importancia en la formación de la competencia profesional; la capacidad de hacer generalizaciones filosóficas basadas en ideas de las ciencias naturales; la capacidad de aplicar los conocimientos teóricos en la práctica para explicar los fenómenos observados y predecir los resultados; profundidad y consistencia del conocimiento; una amplia gama de intereses; actitud consciente hacia el área estudiada; deseo y capacidad de defender su punto de vista profesional; comprender la importancia de la actividad científica para el desarrollo de un campo profesional específico.

La investigación incluye el estudio de la dinámica de participación de los estudiantes en diversas competencias de carácter social, competencias creativas, competencias de habilidades profesionales, lo que muestra el deseo y capacidad de los estudiantes de utilizar la experiencia e ideas de la cosmovisión en actividades profesionales.

Se realiza la valoración de la influencia de la formación de la cosmovisión científica sobre la futura actividad profesional de los estudiantes. 
Los resultados de la investigación obtenidos se someten a análisis cuantitativos y cualitativos. Los estudiantes se caracterizaron en tres direcciones (que se correlacionan con los niveles de formación de la cosmovisión científica): intelectual, emocional y comunicativa. La dirección intelectual contiene el desarrollo del pensamiento crítico, el ingenio, la capacidad de análisis operativo y conclusiones. La dirección emocional incluye confianza en las decisiones tomadas, tranquilidad en situaciones de conflicto, disposición para resolver situaciones difíciles. La dirección comunicativa revela la capacidad de cooperación e interacción efectiva.

Hemos registrado los indicadores de varios grupos de estudiantes para 2018, 2019 y 2020.

\section{Resultados y discusión}

Analizamos el contenido de las disciplinas académicas que aseguran la formación de la capacidad de los estudiantes para utilizar los fundamentos del conocimiento filosófico y socio-humanitario para formar una cosmovisión científica. El estudio involucró a estudiantes de instituciones de educación superior.

En el proceso de aprendizaje se utilizaron tecnologías y métodos modernos que amplían las oportunidades de formación de los estudiantes. El estudio de la filosofía determina como meta la creación de condiciones para el estudio de los fundamentos del conocimiento filosófico y su uso en la formación de una cosmovisión científica (Davydova, 2020). La implementación de tecnologías de aprendizaje por discusión y tecnologías de juegos tiene una influencia activa en el proceso de aprendizaje. El estudio de las secciones "Filosofía, su asignatura y lugares en la cultura", "Historia de la filosofía" y "Fundamentos de la filosofía general" se acompaña de la inclusión de los estudiantes en una interacción activa. Participan en diversas disputas sobre cuestiones problemáticas que surgen en las condiciones modernas, participan en la resolución de problemas contextuales (Loginova et al., 2012) Las fuentes de literatura filosófica que ofrecen los profesores permiten a los estudiantes dominar las obras fundamentales de diversos autores. Además de resolver problemas prácticos, participar en discusiones, los estudiantes forman la habilidad del pensamiento crítico y una cosmovisión científica.

El trabajo independiente en el curso contribuye al desarrollo de la creatividad, las habilidades de investigación, la iniciativa y la capacidad de autoorganización de los estudiantes. Al completar las tareas, los estudiantes estudian tanto el material teórico ubicado en el curso de e-learning como las publicaciones en revistas (Pichugina et al., 2019). Los estudiantes someten el material estudiado a análisis y procesamiento creativo para su discusión en clases prácticas. El desarrollo de la disciplina también se realiza en un entorno electrónico. El control se organiza a través de asignaciones y pruebas en LMS Moodle.

Los estudiantes prueban los conocimientos adquiridos en el proceso de actividades de investigación, participación en diversas conferencias, concursos creativos, proyectos sociales, concursos de habilidades profesionales. En el proceso de enseñanza de la disciplina, se utilizan tecnologías educativas modernas, que permiten a los estudiantes dominar la cantidad necesaria de conocimiento sobre cuestiones filosóficas, identificar rápidamente las características de los fundamentos de la cosmovisión de varias tendencias filosóficas y determinar su importancia en el mundo real. El juego, las tecnologías de diseño implementadas por los profesores contribuyen al desarrollo del pensamiento creativo de los estudiantes, la formación de una visión sistémica de los fenómenos de la vida social y profesional.

La culturología tiene como objetivo revelar los patrones, las formas de funcionamiento de la cultura en todas las etapas del desarrollo histórico y en todas las esferas de la vida humana (Ponachugin et al., 2019). En el curso del estudio de la disciplina, el estudiante domina la dinámica de los procesos socioculturales, la originalidad de la cultura de Rusia y su lugar en la civilización mundial. 
En el proceso de estudiar la estructura y composición del conocimiento culturológico moderno, se utilizan métodos de investigación culturológica y tecnologías de aprendizaje colectivo. Los estudiantes se unen en subgrupos para resolver conjuntamente un problema común, estudiar estudios culturales como ciencia y como objeto de investigación. Los estudiantes llevan a cabo discusiones, participan en tormentas de ideas y mesas redondas, discuten problemas científicos, dominan independientemente el material sobre temas socioculturales, estudian la imagen cultural del mundo, lo que ayuda a formar la capacidad de utilizar los fundamentos del conocimiento filosófico y socio-humanitario para formar una cosmovisión científica.

En las clases de sociología, los estudiantes se forman ideas sobre las leyes de formación, funcionamiento y desarrollo de la sociedad, la capacidad de evaluar los procesos complejos de la realidad social. En el proceso de preparación, se utilizan tecnologías modernas de información y comunicación, que permiten a los estudiantes completar tareas de forma remota e interactuar eficazmente entre sí. Al estudiar el tema "Comunicación social y opinión pública", los estudiantes analizan una gran cantidad de información, seleccionan de forma independiente el material más relevante necesario para la implementación de proyectos grupales e individuales. Los profesores utilizan el método de estudio de casos para revitalizar las experiencias existentes de los estudiantes. Para lograr rápidamente resultados positivos, se utiliza un enfoque individual, teniendo en cuenta las características individuales de los estudiantes.

En el proceso de aprendizaje, los estudiantes dominan los temas debatibles modernos de la naturaleza científica del conocimiento, los fundamentos conceptuales filosóficos y metodológicos de la naturaleza científica del proceso cognitivo a través de la participación activa en actividades de investigación como parte integral de la preparación para la futura profesión.

En la preparación de los estudiantes se implementan activamente tecnologías de aprendizaje contextual, lo que permite crear condiciones cercanas a las profesionales. Para adaptar a los estudiantes a la resolución de problemas y tareas profesionales, se utiliza el método de situaciones problemáticas.

Hemos identificado los criterios e indicadores de la formación de la cosmovisión científica de los estudiantes.

La Tabla 1 muestra los niveles de formación de la cosmovisión científica según los criterios seleccionados.

\begin{tabular}{|l|l|}
\hline Nivel & Característica \\
\hline Alto & $\begin{array}{l}\text { Conocimiento seguro del aparato terminológico; desarrolló la capacidad de hacer } \\
\text { generalizaciones filosóficas basadas en ideas de ciencias naturales; la capacidad de } \\
\text { aplicar los conocimientos teóricos en la práctica; el estudiante demuestra un } \\
\text { conocimiento profundo y sistemático de mí; actitud consciente hacia el área estudiada; } \\
\text { el alumno muestra el deseo y la capacidad de defender su punto de vista profesional; } \\
\text { comprender la importancia de la actividad científica para el desarrollo del campo } \\
\text { profesional elegido. }\end{array}$ \\
\hline Medio & $\begin{array}{l}\text { El alumno posee el aparato terminológico, es capaz de reproducirlo; capaz de } \\
\text { generalizaciones filosóficas m basadas en ideas naturales; sabe aplicar los } \\
\text { conocimientos teóricos en la práctica; demuestra un conocimiento profundo, en parte } \\
\text { no sistemático; muestra una actitud consciente hacia el área estudiada; el alumno } \\
\text { muestra el deseo y la capacidad de defender su punto de vista profesional; consciente } \\
\text { de la importancia de las actividades de investigación para el desarrollo del campo } \\
\text { profesional elegido. }\end{array}$ \\
\hline Bajo & $\begin{array}{l}\text { El alumno utiliza poco el aparato terminológico, reproduce algunos de sus elementos; } \\
\text { incapaz de generalizaciones filosóficas sobre la base de ideas científicas naturales; }\end{array}$ \\
\hline
\end{tabular}


capaz de aplicar los conocimientos teóricos en la práctica; demuestra conocimiento fragmentario; no mostrar conciencia de la relación del campo de estudio; el alumno tiene un deseo débilmente expresado y capacidad para defender su punto de vista profesional; no es consciente y no busca darse cuenta de la importancia de las actividades de investigación para el desarrollo del campo profesional elegido.

Cuadro 1. Criterios para la formación de la cosmovisión científica de los estudiantes de las instituciones de educación superior.

La Figura 1 muestra la dinámica de la formación de la cosmovisión científica de los estudiantes de las instituciones de educación superior.

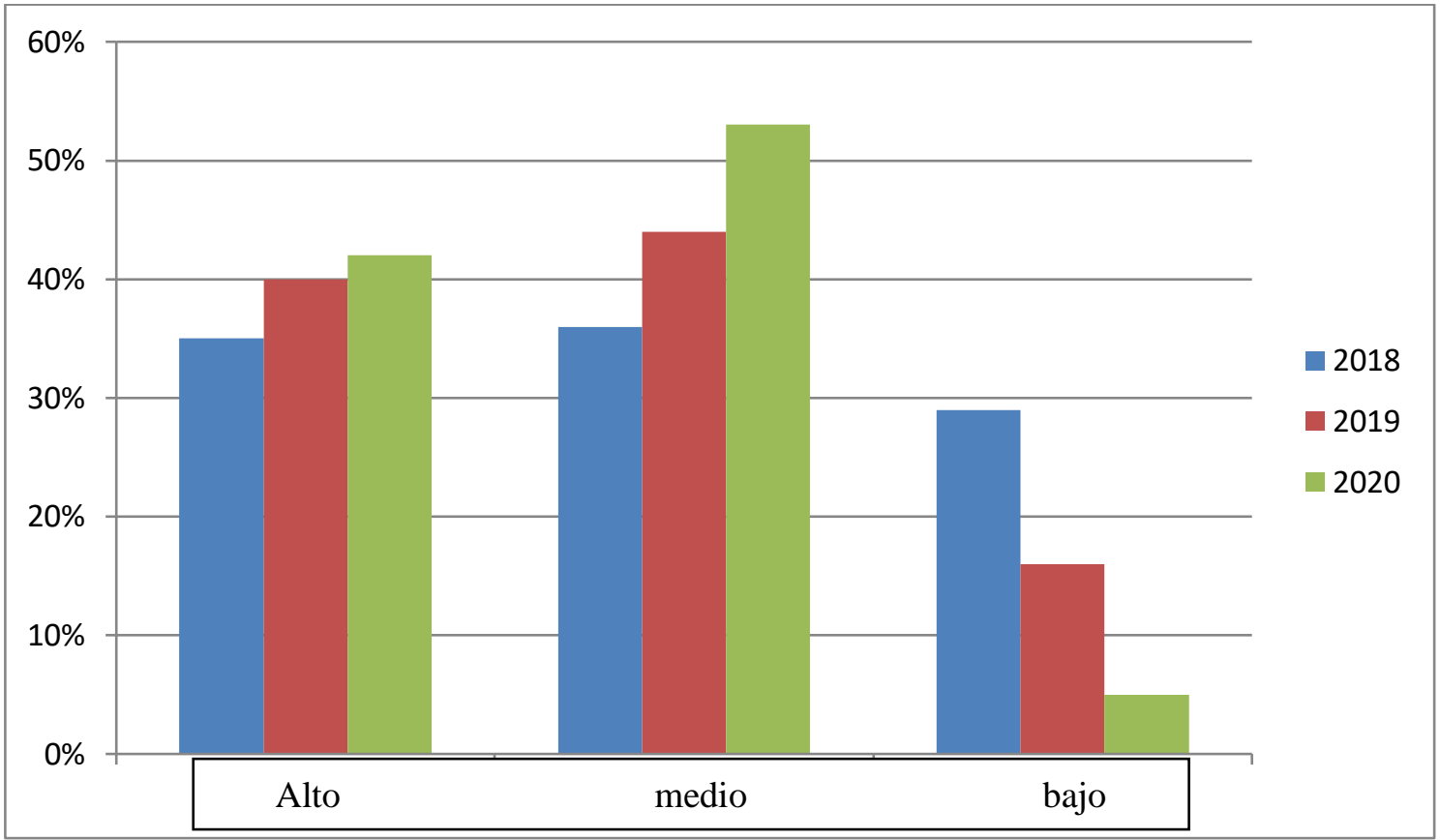

Fig. 1. Resultados del procesamiento estadístico de datos para identificar el nivel de formación de la cosmovisión científica de los estudiantes (como parte de nuestra investigación)

Los criterios presentados no son exhaustivos, pero sí suficientes para determinar la formación de la perspectiva científica de los estudiantes y su capacidad para tomar sus puntos de vista al resolver diversos problemas educativos.

El proceso de formación de una cosmovisión científica incluye la participación de los estudiantes en concursos de trabajos científicos, varios concursos de habilidad profesional. Hemos identificado la dinámica de participación de los estudiantes en diversas competencias profesionales durante varios años. Los resultados se muestran en la Figura 2. 


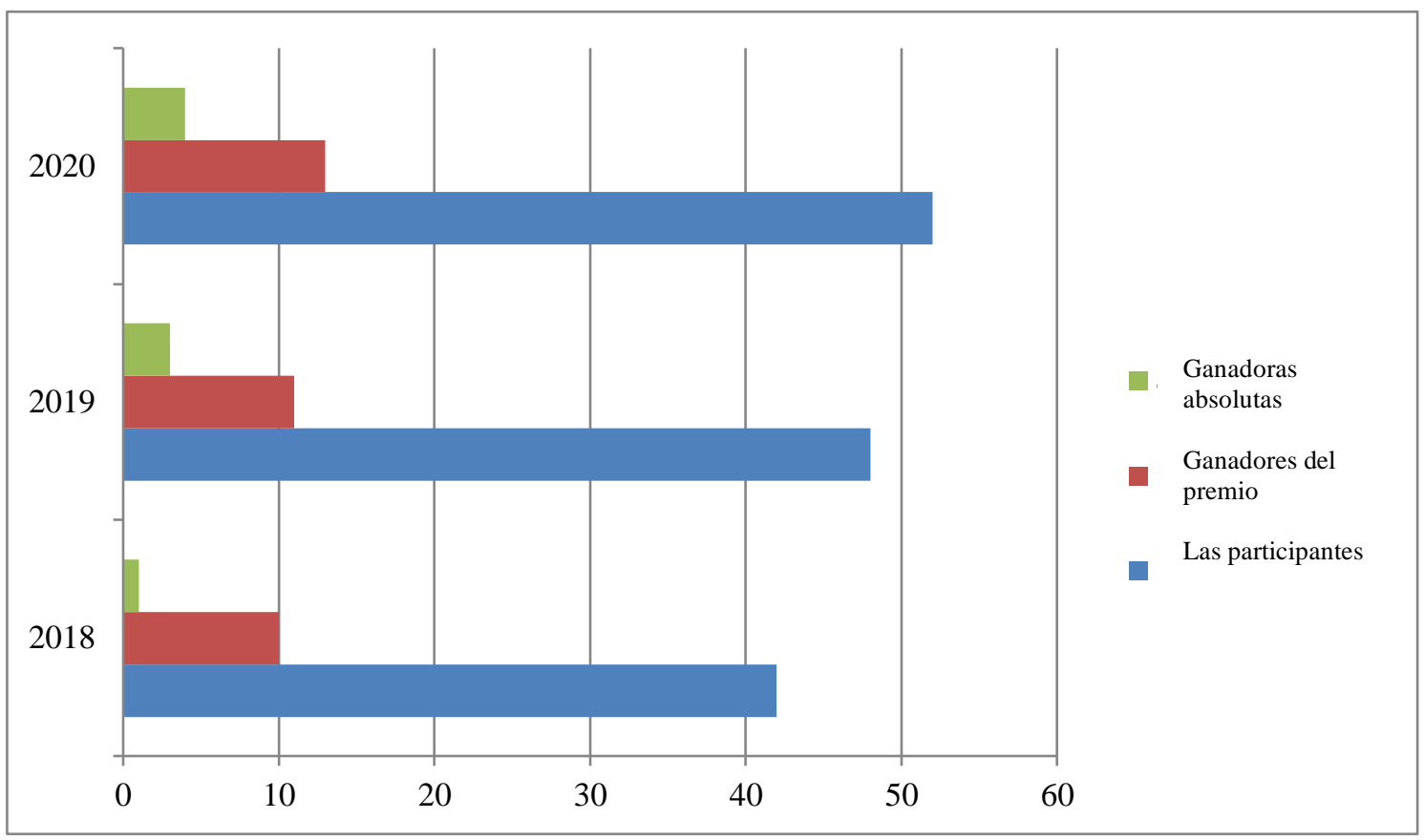

Fig. 1. Dinámica de participación de los estudiantes en competencias de competencias profesionales de varios niveles para el período de 2018 a 2020 (como parte de nuestra investigación)

Los estudiantes comenzaron a unirse en grupos de proyectos, para participar en varias competencias profesionales que amplían sus posiciones científicas y de cosmovisión. La dinámica observada muestra un aumento en el número de postores.

Los resultados del estudio nos permiten hablar de una dinámica positiva pronunciada de la formación de la cosmovisión científica de los estudiantes de instituciones de educación superior.

\section{Conclusiones}

El estudio revela una imagen de la formación de la perspectiva científica de los estudiantes sobre los cambios positivos. Los estudiantes dominan los conceptos básicos de la cosmovisión y amplían gradualmente sus ideas sobre los conceptos existentes, formando su cosmovisión científica a través del estudio de disciplinas relevantes utilizando tecnologías educativas innovadoras.

El estudio permitió establecer dinámicas positivas en la formación de la capacidad de los estudiantes para utilizar los fundamentos del conocimiento filosófico y socio-humanitario y aumentar el nivel de formación de la cosmovisión científica de los estudiantes.

Cada año los estudiantes muestran un gran interés por la actividad científica, muestran una mayor disposición para la actividad profesional.

\section{Referencias bibliográficas}

Arbeláez-Campillo, D.F., \& Villasmil Espinoza, J. J. (2020). Escenarios prospectivos de un nuevo orden internacional que se vislumbra luego de la pandemia COVID-19." Revista Telos, No. 22 (3), Pp. 494. https://doi.org/10.36390/telos223.02 
Arbeláez-Campillo, D., \& Rojas-Bahamon, M. (2020). Pandemics in globalization times. Amazonia Investiga, 9(27), 3-4. https://doi.org/10.34069/AI/2020.27.03.0

Barsukova, N.K., \& Shishelova, T.I. (2008). One of the approaches to the formation of the scientific world view of students // Modern problems of science and education. 4

Birzhenyuk, G.M., \& Efimova, T.V. (2020). "Schering as a new landmark in education". In: Innovative development of vocational education. Vol. 2, No. 26, pp. 12-21.

Chertovskikh, O.O. (2019). "Prospects for the use of digital resources in education". In: Baltiyskiy gumanitarnyy zhurnal (Baltic Humanitarian Journal). Vol. 8, No. 4 (29), pp. 184-187. (in Russ.).

Davydova, T.E. (2020). "The specifics of the organization of the educational process at the university in non-standard conditions". In: Production organizer. Vol. 28, No. 2, pp. 97107.

Donetskova, O. YU. (2019). "Modernization of the modern education system in Russia". In: Baltiyskiy gumanitarnyy zhurnal (Baltic Humanitarian Journal). Vol. 8, No. 2 (27), p.p 37-39. (in Russ.).

Eliseeva, D., Fedosov, A., Agaltsova, D., Mnatsakanyan, O., \& Kuchmezov, K. (2020). The Evolution of Artificial Intelligence and the Possibility of its Application in Cyber Games. Amazonia Investiga, 9(28), 123-129. https://doi.org/10.34069/AI/2020.28.04.15

Filchenkova, I.F. (2019). "Educational management of innovative activity of teachers as an object of pedagogical research". In: Vestnik Mininskogo universiteta (Vestnik of Minin University). Vol. 7, (4), 3. (in Russ.).

Grigoriev, S. G., Shabunina, V. A., Tsarapkina, JU. M., \& Dunaeva, N. V. (2019). "Electronic library system as a means of self-development of students of digital generation $\mathrm{Z}$ (on the example of studying the course "Basics of the counselor activity")". In: Scientific and technical libraries. No. 7. Pp. 78-99. 29.

Ivanov, M., Parnikova, T., Gulyaev, V., \& Petrov, N. (2020). The activity approach implementation in the formation of students' general technical competencies. Amazonia Investiga, 9(26), 205-210. https://doi.org/10.34069/AI/2020.26.02.23

Kalinkina, E.G., \& Gorodetskaya, N.I. (2017). "Development of e-learning and distance learning technologies in the process of professional development of teachers". In: Nizhny Novgorod education. No. 1, pp. 131-138.

Kosikhina, T.V. (1985). Pedagogical conditions for the formation of the dialectical-materialistic worldview of students in the process of studying general scientific disciplines specialty 13.00.01 "General pedagogy, history of pedagogy and education" / T.V. Kosikhina. Saransk, 1985.

Loginova, O. A., \& Ustinskaya, A. (2012). Sociocultural and psychological bases of the educational influence of the collective on the personality of the university graduate. University education collection of articles of the XVI International Scientific and Methodological Conference. Dedicated to the 150th anniversary of the birth of P.A.Stolypin. ed. V.I. Volchikhin, R.M. Pecherskaya. 280-282.

Lutfullaev, G. A., Lutfullaev, U. L., Kobilova, S. S., \& Neymatov, U. S. (2020). Experience of distance learning in the context of the COVID-19 pandemic. Problems of pedagogy, No.4 (49), pp. 66-69. 
Maslennikova, Yu.V., \& Grebenev, I.V. (2013). Formation of the natural-scientific worldview of students of humanitarian educational institutions: Monograph. N. Novgorod: Publishing house of the Nizhny Novgorod University named after N.I. Lobachevsky. 148 p.

Orlova, I.G., \& Lukashik, A.F. (2018). "Distance learning as a way to implement advanced training of specialists in the fuel and energy complex (on the example of FGAOU DPO "KEMRIPK")". In: Bulletin KEMRIPK. No. 2, pp. 46-49.

Petrichev, P. V., Masyuk, N. N., \& Bushueva, M. A. (2018). "Method of estimation of the effectiveness of the partnership russian universities with foreign educational organizations". In: Azimut nauchnykh issledovaniy (Azimuth of Scientific Researches: Economics and Management). Vol.7, No. 3 (24), p.p 229-232.

Pichugina, G.A., \& Bondarchuk, A.I. (2019). "Structure of the training case in the organization of the educational process". In: Humanitarian Balkan Research. Vol. 2, No. 4, pp. 5-7.

Pinkovetskaia, I., Arbeláez-Campillo, D., Rojas-Bahamón, M., \& Veas Iniesta, D. (2020). Motivation of new entrepreneurs in modern economies. Amazonia Investiga, 9(29), 368373. https://doi.org/10.34069/AI/2020.29.05.41

Ponachugin, A.V., Lapygin, YU.N. (2019). "Digital educational resources of the university: design, analysis and expertise". In: Vestnik Mininskogo universiteta (Vestnik of Minin University). Vol. 7 (2), 5. (in Russ)

Tishchenko, A.S. (2020). "Assessment of the losses of educational organizations from a reduction in the volume of additional educational services to the population during a pandemic". In: Economic Development of Russia. Vol. 27, No. 6, pp. 73-79.

Veselova, E.A. (2008). Formation of the scientific outlook of students in the educational process of higher education: abstract for the degree of candidate of pedagogical sciences: specialty 13.00.01 "General pedagogy, history of pedagogy and education" / E.A. Veselova. - Nizhny Novgorod, 2008.

Yakovleva, E. V. (2019). Formation of the scientific outlook of students in laboratory classes in physics at the university. Bulletin of Science and Practice, 5 (4), 398-404. 